\title{
The potential use of Apgar score and neonatal encephalopathy in registry based studies
}

\author{
Dag Moster ${ }^{1,2}$ and Trond Markestad ${ }^{3,2}$ \\ 1) Locus of Registry Based Epidemiology, Department of Public Health and Primary Health Care, \\ University of Bergen, NO-5020 Bergen, Norway \\ 2) Department of Pediatrics, Haukeland University Hospital, NO-5021 Bergen, Norway \\ 3) Section for Pediatrics, Department of Clinical Medicine, University of Bergen, NO-5020 Bergen, Norway \\ Correspondence: Dag Moster, Locus of Registry Based Epidemiology, Department of Public Health and Primary Health Care, \\ P.O. Box 7804, NO-5020 Bergen, Norway \\ Telephone: +4753204027 / +4755975596 Telefax: +4753204001 E-mail: Dag.Moster@smis.uib.no
}

\begin{abstract}
We have previously described the impact of Apgar scores on later major and minor disabilities. According to these and other studies, children with low Apgar scores have to show symptoms of neonatal encephalopathy in the first week of life to be at risk of developing later disabilities. Information on neonatal encephalopathy has traditionally been less accessible than Apgar scores in The Medical Birth Registry of Norway (MBRN). In the present study the possibility of extracting information on neonatal encephalopathy from the new notification form to the MBRN, which was introduced in late 1998, was investigated. The introduction of specific and generally accepted definitions of neonatal diagnoses combined with more comprehensive reports to MBRN from the neonatal departments may render registry based studies on neonatal encephalopathy possible in the future.
\end{abstract}

\section{INTRODUCTION}

The first clinical examination we face in life is the Apgar score which is usually performed at 1 and $5 \mathrm{mi}-$ nutes after birth and repeated every five minute if the score is low. The score has been described as a standardized mini-examination of brainstem function (1) based on five clinical signs: heart rate, respiratory effort, reflex irritability, muscle tone and colour. Each of these items is rated with the three response options, 0 , 1 and 2, and all are added to comprise the Apgar score, which can range from 0 to 10 . A score of 7-10 is considered to be normal (2).

The Apgar score was originally proposed by the anaesthesiologist Virginia Apgar in 1953 (3) and gained rapid worldwide use, but its value has repeatedly been questioned. Concerns have been raised regarding its reliability, its ability to discriminate between good and poor condition at birth, its association with intrauterine asphyxia and its predictive value for short and long term outcome $(2,4-6)$.

While the Apgar score only describes the condition during the first minutes of life, neonatal encephalopathy (NE) is a clinical syndrome describing cerebral symptoms in the earliest days of life in the term infant. The term was described by Sarnat and Sarnat in 1976 and is manifested by difficulty with initiating and maintaining respiration, depression of tone and reflexes, subnormal level of consciousness and often seizures (7-9).

Since the Apgar score is used world-wide and is easily performed and recorded in large data sets, it has often been used as outcome measure in studies of perinatal care. NE may seem to be more difficult to record since the diagnosis is often set during a time span of several days and has been defined differently by clinicians (9). In perinatal research, however, it is of great interest to identify newborns with NE as it is now accepted that the pathway from an intrapartum hypoxic-ischemic injury to subsequent cerebral palsy must progress through NE (1).

We have earlier evaluated the potential use of Apgar score as outcome measure in studies of perinatal care $(10,11)$. In these papers we also had the opportunity to study the impact of NE in a smaller cohort. In the present paper we provide a brief review of these papers and discuss the possibility of extracting information on NE from the Medical Birth Registry of Norway (MBRN). The latter may be possible after the revised notification form was introduced in 1998. With this revision a more comprehensive report of diagnoses is provided from the neonatal departments to MBRN.

\section{MATERIAL AND METHODS}

The present paper is based on a review of previous studies which we have published on significance of Apgar scores with respect to survival and neurodevelopmental outcome $(10,11)$, and analyses of data based on notifications to the MBRN after the introduction of a new notification form in 1998 which contains more information on neonatal morbidity, including cerebral function. 
In the first paper (10), all 235165 children born in Norway between 1983 and 1987 with a birthweight of at least 2500 grams and with no congenital anomalies were identified in the MBRN and followed from birth until 8 to 12 years of age by linking the MBRN data with two other national registers. From the Death Register we received information on death and time of death, and from The National Health Insurance Scheme information on chronic disabilities. This cohort was used to assess the impact of Apgar sores on death and neurologic diagnoses.

Furthermore, from the original cohort we identified a subcohort that comprised all survivors with a fiveminute Apgar score of 0-3 and a random sample of the other scores, a total of more than 1000 children. All infants with a five minute score of $0-6$, later registered as dead were also included.

A 117-item questionnaire was mailed to the parents of all survivors in this subcohort when the children were 8 to 13 years old. Parents were asked about conditions during birth, the newborn period, later diagnoses, learning difficulties, school performances, behavioural items and need for added resources in kindergarten or at school. Parents were also asked for written permission to collect discharge summaries from hospitals. The Norwegian Board of Health gave permission to collect hospital discharge summaries for the children who died.

The comprehensive information obtained for this subcohort had three purposes. First, it was used to validate the information on chronic disabilities given by the The National Health Insurance Scheme in the large cohort (10). Second, it made it possible to expand the information from the newborn period with information on symptoms of NE $(10,11)$ and third, minor sequelae among children without major chronic disabilities could be assessed (11).

In these studies, a child was classified as having had symptoms of NE if he or she during the first week of life was ventilated beyond the initial need for resuscitation, had seizures or had feeding problems. Among the parents of the surviving children, $76 \%$ answered the questionnaire adequately.

In the second paper we studied the rate of minor disabilities according to Apgar score after excluding children with severe disabilities or congenital anomalies registered either at the MBRN or the National Insurance Scheme, or reported in discharge summaries or by the parents. Thus 727 children were selected (11).

In addressing the possibility of relating Apgar scores with severity of NE we searched the MBRN records for all liveborn infants without congenital anomalies and with a birthweight of at least 2500 gram born in the years 1999-2004. Apgar scores at one and five minutes and diagnoses related to NE were identified.

Sarnat and Sarnat described a classification of neonatal encephalopathy in three stages (7), and this staging is still used by clinicians. The International Classification of diseases' tenth revision (ICD 10) has no specific diagnosis that describes these stages, but the Norwegian Pediatric Society has recently suggested to use the code P91.3 - Neonatal cerebral irritability for stage 1, the code P91.4 Neonatal cerebral depression for stage 2 and the code P91.5 Neonatal coma for stage 3 (12).

The MBRN now records ICD10 diagnoses set by the neonatal departments, and it is also possible to mark the diagnoses cerebral irritability or cerebral depression directly in separate boxes on the new notification form.

Newborns recorded with the ICD10 code P91.3 or with cerebral irritability were considered to have NE grade 1, while we found it more difficult to distinguish between grade 2 and 3 and grouped children with ICD10 code P91.4 or P91.5 or with cerebral depression as $\mathrm{NE}$ grade2/3.

\section{RESUlts}

Among children born in Norway in the years 19831987 without congenital anomalies and with normal birthweights, only $0.1 \%$ had a five minute Apgar score of $0-3$, and $0.6 \%$ a score of $4-6$. The distribution of the five-minute score has been fairly constant during the years it has been recorded.

Low Apgar scores were associated with neonatal death and cerebral palsy, but less convincingly with risk of developing other chronic disabilities. Children with a five-minute score of $0-3$ had an 81-fold increased risk of cerebral palsy and a 9-fold risk of mental retardation without cerebral palsy, but not a significantly increased risk of non-neurological disabilities (10). If the Apgar score was below 4 both one and five minutes after birth, surviving children had a 145-fold increased risk of developing cerebral palsy compared to children who had normal scores for both.

The validation of the National Insurance Scheme using information from the subcohort, demonstrated that the majority of children with cerebral palsy were correctly recorded. Furthermore, all the children in the subcohort with low Apgar scores who developed cerebral palsy also had symptoms of NE in the first week of life (10).

The same was true when children with major handicaps were excluded. The combination of a low fiveminute Apgar score and symptoms of NE in the first week of life implied a significant increased risk of both learning and behavioural disorders compared to children with normal scores and no NE (11). However, no significant increased risk could be demonstrated for most of the outcomes studied if only one of these items, i.e. Apgar score or signs of NE, was pathological.

In the years 1999-2004, cerebral irritability was recorded for 2.2 per thousand births, and cerebral depression for 0.5 per thousand (Table 1). No children 
were recorded with a diagnosis of neonatal coma. Both cerebral irritability and cerebral depression had a strong association with low Apgar scores (Table 2).

\section{DISCUSSION}

In the first paper, we demonstrated a very strong association between low Apgar score and later cerebral palsy with as much as an 81 -fold increased risk if the five minute score was below 4 and a 145 -fold increased risk if the scores at both one and five minutes were in the range $0-3$. It is unusual to find a marker in the neonatal period that is associated with an increased risk of a later chronic disability of that magnitude. However, the clinical consequences of these findings might not be so obvious. Most children who later develop cerebral palsy will not have low scores and most children with low scores do not develop cerebral palsy.

The two studies demonstrated, however, that the ability to predict later adverse outcome was increased when information on NE was combined with information on Apgar scores. This was true both for cerebral palsy and learning and behavioural disabilities.

Table 1. Distribution of neonatal encephalopathy (NE) total number (per thousand births) among 316073 liveborn infants with birthweight $\geq 2500$ grams and no congenital anomalies born in 1999-2004.

\begin{tabular}{lcc}
\hline Year of birth & NE Grade 1* & NE Grade 2 and 3** \\
\hline 1999 & $119(2.2)$ & $34(0.6)$ \\
2000 & $117(2.1)$ & $14(0.3)$ \\
2001 & $95(1.8)$ & $24(0.5)$ \\
2002 & $120(2.3)$ & $27(0.5)$ \\
2003 & $107(2.1)$ & $25(0.5)$ \\
2004 & $139(2.7)$ & $20(0.4)$ \\
\hline
\end{tabular}

* Reported to MBRN with "cerebral irritation" or ICD10 code P91.3 - Neonatal cerebral irritability

** Reported to MBRN with cerebral depression or ICD10 code P91.4 - Neonatal cerebral depression

Table 2. Relationship between five-minute Apgar score and neonatal encephalopathy (NE) among 315234 liveborn infants with birthweight $\geq 2500$ grams and no congenital anomalies born 1999-2004

\begin{tabular}{lccr}
\hline & NE grade 2/3 & NE grade 1 & \multicolumn{1}{c}{ Without NE } \\
\hline Apgar 0-3 & $33(7.5 \%)$ & $54(12.2 \%)$ & $354(80.3 \%)$ \\
Apgar 4-6 & $46(2.1 \%)$ & $143(6.4 \%)$ & $2036(91.5 \%)$ \\
Apgar 7-10 & $65(0.02 \%)$ & $497(0.2 \%)$ & $312006(99.8 \%)$ \\
\hline
\end{tabular}

An English study demonstrated that $80 \%$ of term infants without congenital malformation who had symptoms of NE, had evidence of perinatally acquired insults (13). It is also generally accepted that a child who develops cerebral palsy after a perinatal event has shown symptoms of neonatal encephalopathy in the first week of life (1). According to our studies, this seems also to be true not only for major disabilities (10), but also for learning and behavioral disabilities (11).

In studies of later outcome after perinatal asphyxia it is therefore mandatory to have information on presence and severity of NE. This diagnosis has not traditionally been recorded by the MBRN, but on the new notification form in use from late 1998, this information may now be possible to extract as demonstrated in the present study.

Neonatal encephalopathy has been estimated to occur in 1-6 per 1000 live term births $(14,15)$. This is consistent with the figures of 2 to 3 per thousand found in the present study. However, the proportion of the most severe grades was much lower than expected. It has been estimated that perinatal asphyxia causing moderate or severe encephalopathy occurs in approximately 2 per thousand births $(16,17)$, while the two most severe grades of NE was only registered for 0.3 0.6 per thousand live term births among those delivered after the introduction of the new notification form to the MBRN. The low figure might be due to better perinatal care in this country, but is more probably due to an underreporting of NE to MBRN. The relatively low proportion $(20 \%)$ of recorded NE among infants with an Apgar score of 0-3 at five minutes also suggests underreporting. Based on retrospective information from parents we have previously found that around $50 \%$ of infants registered with a five minute Apgar score of 0-3 had symptoms indicative of neonatal encephalopathy (11).

The reported incidence of various grades of NE clearly demonstrates the problems of using a clinical term that probably has been defined and reported differently by different neonatal departments. It is promising that the Norwegian Pediatric Society now has suggested specific ICD-10 codes to be used nationwide for the different grades of NE (12). Together with an increased focus on reporting correct and reliable diagnoses from the neonatal departments to MBRN $(18,19)$ these factors are expected to increase the reliability of future national registry based studies of perinatal asphyxia.

\section{REFERENCES}

1. The American College of Obstetricians and Gynecologists and American Academy of Pediatrics. Neonatal Encephalopathy and Cerebral Palsy: Defining the Pathogenesis and Pathophysiology. Washington: ACOG, 2003.

2. The Apgar score. Pediatrics 2006; 117 (4): 1444-7. 
3. Apgar V. A proposal for a new method of evaluation of the newborn infant. Curr Res Anesth Analg $1953 ; 32$ (4): 260-7.

4. Schmidt B, Kirpalani H, Rosenbaum P, Cadman D. Strengths and limitations of the Apgar score: a critical appraisal. J Clin Epidemiol 1988; 41 (9): 843-50.

5. Is the Apgar score outmoded? Lancet 1989; 1 (8638): 591-92.

6. Marlow N. Do we need an Apgar score? Arch Dis Child 1992; 67 (7 Spec No): 765-7.

7. Sarnat HB, Sarnat MS. Neonatal encephalopathy following fetal distress. A clinical and electroencephalographic study. Arch Neurol 1976; 33 (10): 696-705.

8. Nelson KB, Leviton A. How much of neonatal encephalopathy is due to birth asphyxia? Am J Dis Child 1991; 145 (11): 1325-31.

9. Leviton A, Nelson KB. Problems with definitions and classifications of newborn encephalopathy. Pediatr Neurol 1992; 8 (2): 85-90.

10. Moster D, Lie RT, Irgens LM, Bjerkedal T, Markestad T. The association of Apgar score with subsequent death and cerebral palsy: A population-based study in term infants. J Pediatr 2001; 138 (6): 798-803.

11. Moster D, Lie RT, Markestad T. Joint association of Apgar scores and early neonatal symptoms with minor disabilities at school age. Arch Dis Child Fetal Neonatal Ed 2002; 86 (1): F16-21.

12. Subgruppen i Nyfødtmedisin Norsk Barnelegeforening. Forslag til enhetlige nasjonale kriterier for bruk av neonatale diagnosekoder i ICD-10. Oslo: Den norske lægeforening, 2006.

13. Cowan F, Rutherford M, Groenendaal F, et al. Origin and timing of brain lesions in term infants with neonatal encephalopathy. Lancet 2003; 361 (9359): 736-42.

14. Gonzalez FF, Miller SP. Does perinatal asphyxia impair cognitive function without cerebral palsy? Arch Dis Child Fetal Neonatal Ed 2006; 91 (6): F454-9.

15. Volpe JJ. Neurology of the newborn. Philadelphia: Saunders, 2000.

16. Levene MI. Management and outcome of birth asphyxia. In: Levene MI, Lilford RJ, Bennett MJ, Punt J, eds. Fetal and Neonatal Neurology and Neurosurgery. London: Churchill Livingstone, 1995: 427-42.

17. UK TOBY Cooling Register Protocol. National Perinatal Epidemiology Unit University of Oxford, 2006. (Accessed May 24., 2007, at http://www.npeu.ox.ac.uk/tobyregister/downloads/Cooling\%20Register\%20 Protocol\%20version\%202_28.11.06.pdf.)

18. Medisinsk fødselsregister. Meldeskjema fra Barneavdelingene. Fødselsnytt 2004 (2).

19. Rønnestad A. Orientering om etablering av Norsk Nyfødtmedisinsk Kvalitetsregister. NeoNytt - Subgruppen for Nyfødtmedisin 2006 (2). 